\title{
Description de la femelle de Phlebotomus (Larroussius) Chadli Rioux, Juminer et GibILY, 1966 (Diptera : PSyChOdIDAe). D’aPRÈs UN EXEMPLAIRE CAPTURÉ AUX ENVIRONS DU Kef (TUNISIE)
}

\author{
CHAMKHI J.*, GUERBOUJ S.*, BEN ISMAIL R.*,** \& GUIZANI I.*
}

Summary: DESCRIPTION OF THE HITHERTO UNKNOWN FEMALE OF Phlebotomus (LARRoussius) CHadLII, Rioux, Juminer \& Gibily, 1966 (Diptera: Psychodidae)

The female of Phlebotomus chadlii Rioux, Jumminer \& Gibily, 1966 is described and illustrated for the first time from a specimen collected in El Kef region, northwest Tunisia. It was distinguished from $P$. ariasi by several characters of the spermathecae: 1) the enlarged portion of $P$. chadlii spermathecae duct appears smooth and better developed than that of $P$. ariasi; 2 ) in P. chadlii, this part comprises three quarters of the duct whereas, in $P$. ariasi, it covers only the half; 3 ) the spermathecae neck of $P$. chadlii is shorter than that of $P$. ariasi. The duct base is compatible with the large aedeagus size of $P$. chadlii male. Besides, the assignment of this female to the species $P$. chadlii is supported by: 1) the presence of males in the same area, over the last three years; 2) the total absence in this area of $P$. ariasi; 3) the concomitant presence, in the same trap station, of the described female with $P$. chadlii males.

KEY WORDS : Sandfly, Larroussius, taxonomy, Tunisia.

\section{Résumé :}

La femelle de l'espèce Phlebotomus (Larroussius) chadlii Rioux, Juminer \& Gibily, 1966, inconnue jusqu'à ce jour, est décrite à partir d'un exemplaire capturé au nord-ouest de la Tunisie, dans la région du Kef. Elle a pu être distinguée de P. ariasi par plusieurs caractères de la spermathèque: 1) chez $\mathrm{P}$. chadlii, la partie évasée du conduit apparaît plus lisse et plus large que chez P. ariasi; 2) chez P. chadlii cette partie représente les trois-quart du conduit alors que chez P. ariasi elle n'en forme que la moitié, 3) chez P. chadlii, le col de la spermathèque est plus court que celui de P. ariasi. La base du conduit est compatible avec la grande taille de la valve pénienne de P. chadlii. Par ailleurs, l'attribution de cette femelle à l'espèce P. chadlii est soutenue par: 1) la présence de mâles P. chadlii dans la même localité, durant trois années successives; 2) l'absence totale de P. ariasi dans cette région; 3) la coexistence, dans la même station, de la femelle décrite, avec des mâles de P. chadlii.

MOTS CLÉS : Phlebotomes, Larroussius, taxonomie, Tunisie.

\section{INTRODUCTION}

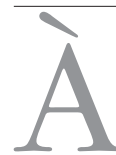

ce jour, la liste des Phlébotomes de la Tunisie comporte 16 espèces : Phlebotomus (Phlebotomus) papatasi (Scopoli, 1786); P. (Paraphlebotomus) sergenti Parrot, 1917; P. (Pa.) alexandri Sinton, 1928; P. (Pa.) chabaudi Croset, Abonnenc et Rioux, 1971; P. (Pa.) riouxi Depaquit, Léger et Killick Kendrick, 1998; P. (Larroussius) ariasi Tonnoir, 1921; P. (L.) chadlii Rioux, Juminer et Gibily, 1966; P. (L.) perniciosus Newstaed, 1911; P. (L.) longicuspis Nitzulescu, 1930; P. (L.) langeroni Nitzulescu, 1930; P. (L.) perfiliewi Parrot, 1930; Sergentomyia (Sergentomyia) antennata Newstaed, 1912; S. (Se.) minuta parroti Adler et Theodor, 1927; S. (Se.) fallax Parrot, 1921; S. (Sintonius) clydei Sinton, 1922 et S. (Grassomyia) dreyfussi Parrot, 1933. En Tunisie, ces espèces sont

* Laboratoire d'Épidémiologie et Écologie Parasitaire, Institut Pasteur de Tunis, BP 74, 1002 Tunis Belvédère, Tunisie.

** Adresse actuelle : CTD/DCD, World Health Organization, Eastern Mediterranean Regional Office, Cairo, Egypt.

Correspondance : Ikram Guizani.

Tél. : 21671843755 ext 540 - Fax : 21671791833.

E-mail : ikram.guizani@pasteur.rns.tn connues, à la fois, par les mâles et les femelles, sauf $P$. chadlii pour qui seul le mâle a été décrit.

Depuis la découverte princeps, dans la région de Gafsa, (Rioux et al., 1966), d'autres échantillons de P. chadlii, toujours de sexe mâle, ont été retrouvés dans le Djebel Boukornine près de Tunis (Croset et al., 1966), dans le Djebel Zaghouan en Kroumirie (Croset et al., 1969), et au Krib près du Kef (Dancesco et al., 1968).

En dehors de Tunisie, $P$. chadlii a été signalée en Algérie (Rioux et al., 1970) et au Maroc (Rioux et al., 1975).

Dans le présent travail, nous proposons la description de la femelle de cette espèce.

\section{MATÉRIEL ET MÉTHODES}

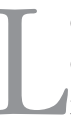

es Phlébotomes étudiés proviennent de la région du Kef (nord-ouest de la Tunisie, étage bioclimatique semi-aride moyen), dans lesquelles une enquête entomologique avait été effectuée de juillet en novembre 2004 pour l'exploration des vecteurs des leishmanioses cutanées à $L$. infantum. L'enquête a porté sur les localités de Oued Souani, située à $15 \mathrm{~km}$ à l'est du 
Kef, et de Bahra, située à $25 \mathrm{~km}$ au nord du Kef. Les phlébotomes ont été capturés aux pièges lumineux CDC, installés, la nuit durant, à l'intérieur des habitations, dans les abris d'animaux (moutons, chèvres, bovins, volailles...) et à l'extérieur, près des chiens. Les récoltes ont été tuées à la fumée de tabac, les mâles montés dans une goutte de liquide de Marc-André, les femelles disséquées extemporanément, à la recherche de leishmanies; les deux derniers anneaux de la spermathèque étant montés dans une goutte de Berlèse pour identification.

Parmi les espèces récoltées (Guerbouj et al., sous presse), trois femelles appartenant au sous-genre Larroussius présentaient des caractères morphologiques proches de
$P$. ariasi. Une première provenait de la localité de Oued Souani (intérieur de maison, 22 juillet 2004); dans cette même station, plusieurs mâles de $P$. chadlii étaient observés en 2001, 2002 et 2003 (Guerbouj et al., sous presse). Par la suite, deux autres femelles étaient capturées à Bahra : un exemplaire dans un abri de chèvres et de moutons (11 août 2004); alors qu'à quelques mètres de la même station, un deuxième exemplaire femelle et un mâle de l'espèce $P$. chadlii étaient récoltés dans un poulailler (12 octobre 2004).

C'est l'une des femelles de Bahra qui a été utilisée pour les mesures morphométriques, les deux autres ayant été endommagées lors de manipulations. À titre comparatif, deux lots de femelles de $P$. ariasi ont été utili-
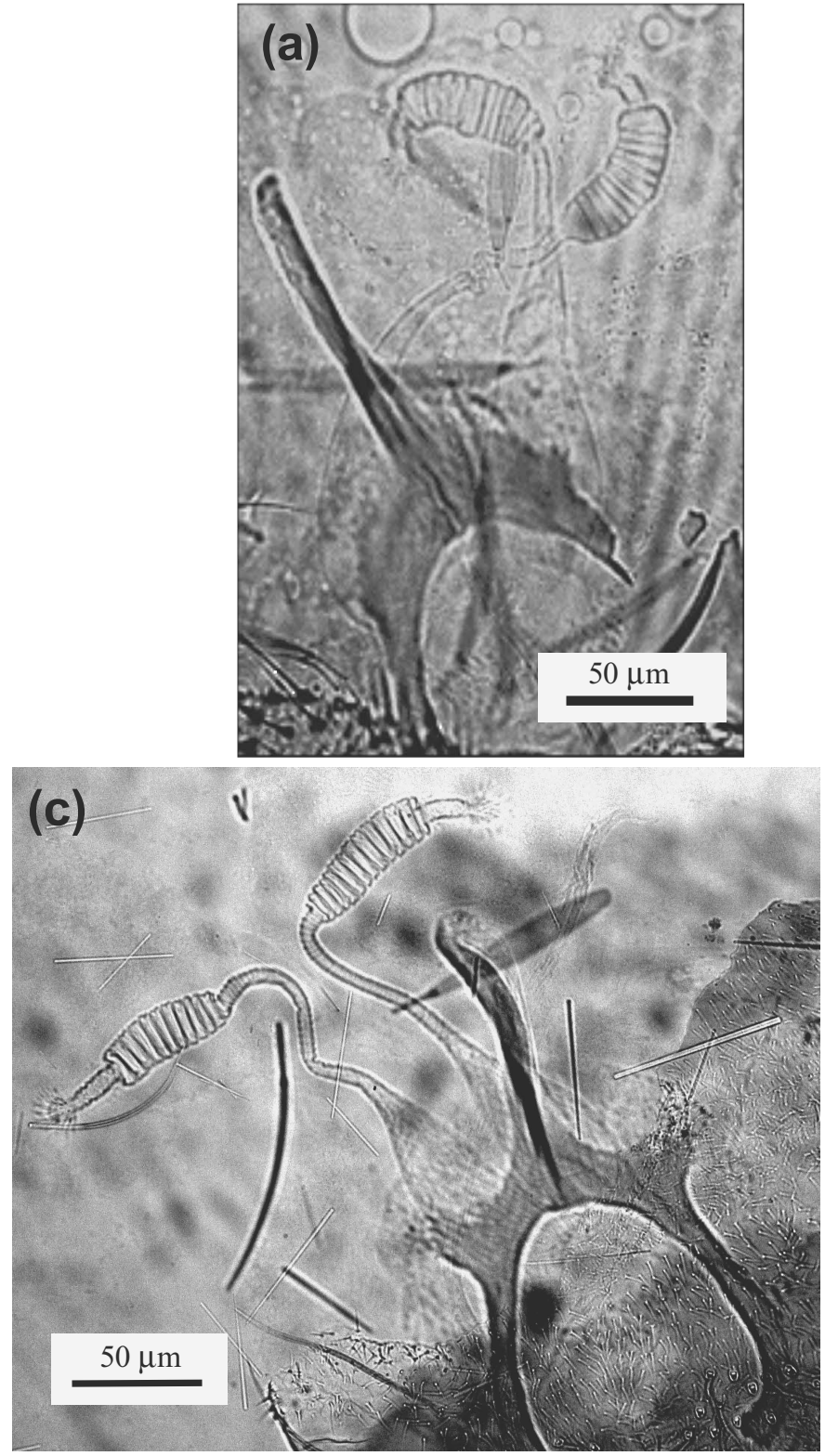
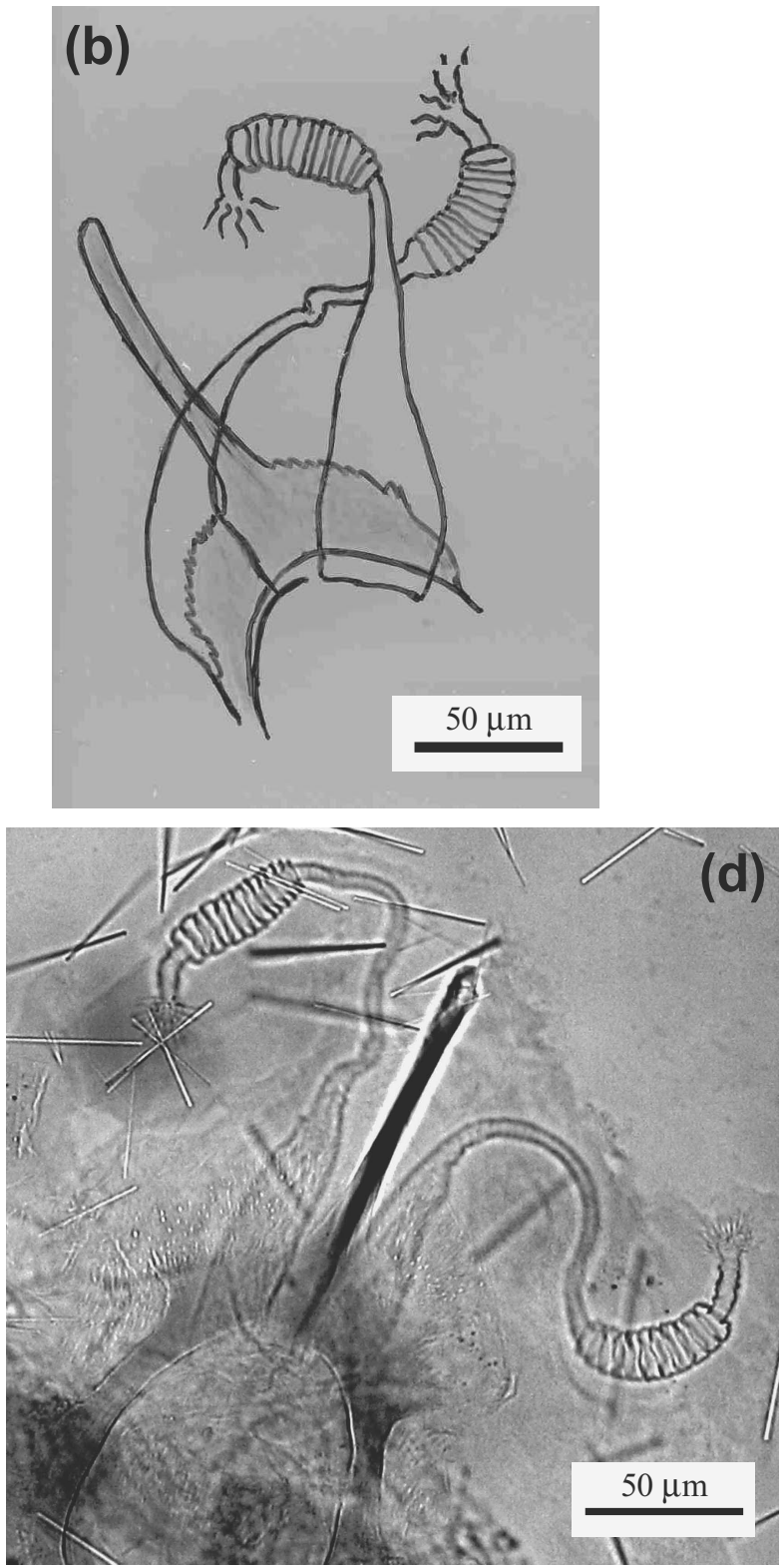

Fig. 1. - Spermathèques de Phlebotomus chadlii, photographiée (a) et dessinée (b) de P. ariasi de France (c) et du Portugal (d). 
sés : quatre provenaient de la région des Cévennes en France et quatre de la région de Cheires au Portugal (mesures réalisées au microscope à objectif micrométrique).

\section{DESCRIPTION}

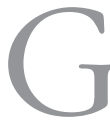
enre Phlebotomus Rondani et Berté, 1840. Sous-genre Larroussius Nitzulescu, 1931. Espèce Phlebotomus chadlii Rioux, Juminer \& Gibily, 1966.

\section{FEMELLE}

Un exemplaire examiné. Taille : $2885 \mu \mathrm{m}$.

- Tête

Taille : $460 \mu \mathrm{m}$.

Cibarium inerme.

Pharynx : longueur $250 \mu \mathrm{m}$. Armature pharyngienne : denticules occupant le $1 / 3$ distal du pharynx.

Formule palpale : 1, 4, 3, 2, 5 .

Formule antennaire : 2/III-IV.

A III $=381,8 \mu \mathrm{m}$. A IV $=$ A V $=151,8 \mu \mathrm{m}$.

A III $>$ A IV + A V.

Épipharynx : 404,8 $\mu \mathrm{m}$. A III/E : 0,943.

c/b : 1,92 (c : $120 \mu \mathrm{m}, \mathrm{b}: 62,5 \mu \mathrm{m})$.

- Thorax

Vestiture du mésanépimère et du mésanépisterne non examinée.

Aile : Longueur : $3100 \mu \mathrm{m}$; largeur : $925 \mu \mathrm{m} ; \alpha$ : $700 \mu \mathrm{m} ; \beta$ : $375 \mu \mathrm{m} ; \gamma: 500 \mu \mathrm{m} ; \delta: 225 \mu \mathrm{m} ; \pi$ : 12,5 $\mu \mathrm{m}$. Largeur de l'aile/ $\gamma$ : 1,85.

- Spermathèques (figure 1)

Corps en manchon, formé de 13 anneaux dont le premier et le dernier sont plus larges que les intermédiaires. Col trapu, représentant $1 / 5$ du corps et se terminant par une tête subarrondie, couverte de soies, légèrement plus large que le col.

Conduits indépendants sur toute leur longueur, lisses, paraissant dépourvus de réticules et présentant une dilatation sur les 3/4 basaux de sa longueur. Longueur totale du conduit $132,5 \mu \mathrm{m}$; sa partie renflée s'élargissant progressivement pour atteindre $37 \mu \mathrm{m}$ de largeur. Mensurations comparées $P$. chadlii/P. ariasi (tableaux I et II).

\section{MÂLE}

Un exemplaire examiné. Morphologie semblable à celle décrite par Rioux et al. (1966).

- Tête

Formule palpale : 1, 4, 2, 3, 5 .

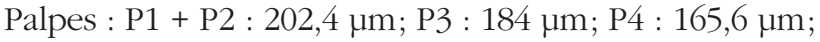
P5 : 423,2 $\mu \mathrm{m}$.

Formule antennaire : 2/III-VIII, 1/IX-XV

A III $=358,8 \mu \mathrm{m}$. A IV $=$ A V $=165,6 \mu \mathrm{m}$.

$\mathrm{A}$ III $>\mathrm{A} \mathrm{IV}+\mathrm{A} \mathrm{V}$.

$\mathrm{c} / \mathrm{b}: 2,789$.

- Thorax

Vestiture du mésanépimère et du mésanépisterne non examinée.

Aile : longueur : $2275 \mu \mathrm{m}$; largeur : $650 \mu \mathrm{m} ; \alpha$ : $500 \mu \mathrm{m}$; $\beta: 300 \mu \mathrm{m} ; \gamma: 375 \mu \mathrm{m} ; \delta: 150 \mu \mathrm{m} ; \pi: 12,5 \mu \mathrm{m}$.

- Armature génitale

Coxite pourvu d'une plage interne de 96 soies. Pénis "en battant de cloche", de $155 \mu \mathrm{m}$ de long sur $25 \mu \mathrm{m}$ de large (dans sa partie renflée).

\section{DISCUSSION}

L 'examen des Phlébotomes femelles capturés dans les localités de Bahra et d'Oued Souani, au nord de 1 la Tunisie, a permis de constater que les formes de la spermathèque et du conduit collecteur se rapprochent de celles de $P$. ariasi. Toutefois, la partie dilatée occupe la moitié de la longueur de ce conduit chez $P$. chadlii alors qu'elle en occupe les trois-quarts chez $P$. ariasi. Autre différence, la brièveté du cou de la spermathèque : le rapport longueurs du cou/corps est de 0,2 pour la femelle tunisienne alors qu'il est de 0,55 pour $P$. ariasi de France et de 0,6 pour $P$. ariasi du Portugal. Les denticules du pharynx occupent le $1 / 3$ chez

\section{Femelle de $P$. arias}

Pharynx

Spermathèque

Col de la spermathèque

Tête du col de la spermathèque Conduits

Partie distale, non dilatée des conduits

Partie évasée des conduits
Denticules occupant le $1 / 5$ du pharynx En forme de manchon, anneaux réguliers Longueur supérieure à la moitié de celle de la spermathèque

Ovalaire

Striées

Occupe la moitié de la longueur totale du conduit

Largeur : $30 \mu \mathrm{m}$

Ornementation en nid d'abeille
Femelle de $P$, chadlii

Denticules occupant le $1 / 3$ du pharynx En forme de manchon, anneaux irréguliers Longueur égale au $1 / 5$ de la spermathèque

Sphérique

Lisses

Occupe les 4/5 de la longueur totale du conduit

Largeur : $37,5 \mu \mathrm{m}$

Lisse

Tableau I. - Caractères différentiels des femelles de Phlebotomus ariasi et $P$. chadlii. 


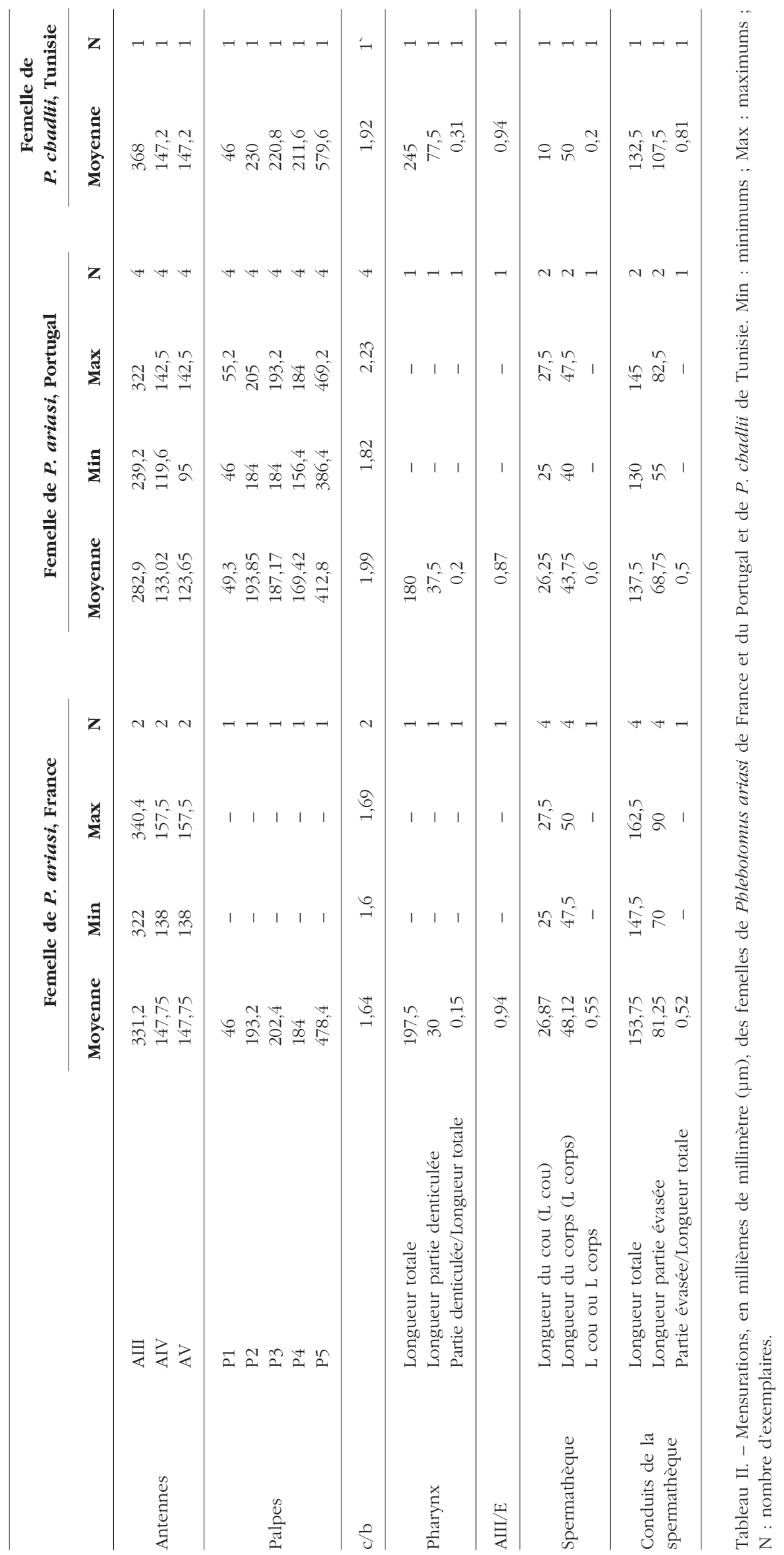


notre spécimen (rapport partie denticulée/longueur totale : 0,31 ), alors qu'ils représentent le $1 / 5$ chez $P$. ariasi de France et du Portugal (rapport partie denticulée/longueur totale : 0,15 et 0,2 , respectivement).

Les mâles de $P$. chadlii ont été capturés dans la même station (Bahra) que le spécimen femelle décrit ci-dessus, et c'est toujours $P$. chadlii mâle qui a été identifié dans les récoltes de la station de Oued Souani, inventoriée durant trois saisons successives. Rappelons que les mâles de $P$. chadlii et $P$. ariasi se distinguent sans difficulté par la forme de la phallothèque et le nombre de soie de la touffe du coxite : 96 chez le premier contre 24 chez le second (Croset et al., 1966). Notons enfin que l'appartenance des deux stations inventoriées, à l'étage bioclimatique semi-aride, concorde avec la répartition de $P$. chadlii et non avec $P$. ariasi que l'on n'observe en Tunisie qu'aux étages humide et subhumide (Croset et al., 1966; Croset, 1969). D'autres captures dans les mêmes sites devraient permettre de compléter la description et de préciser la distribution statistique des caractères diagnostiques.

\section{REMERCIEMENTS}

N ous remercions Dr J. Depaquit, Laboratoire de Parasitologie, Faculté de Pharmacie, Reims, France, et Pr M.O. Afonso, Unidade de Entomologia Médica, Instituto de Higiene e Medicina Tropical, Lisboa, Portugal, pour les échantillons de $P$. arias $i$ femelle qu'ils nous ont aimablement fournis. Nous remercions également les Prs J.A. Rioux, R. Killick-Kendrick, M. Killick-Kendrick, N. Léger et S. Boubaker pour leurs conseils. Le travail sur le terrain a bénéficié d'un financement du MRSTDC-Tunisie dans le cadre des contrats de programmes de recherche.

\section{RÉFÉRENCES}

Croset H., Rioux J.A., Juminer B. \& Tour S. Présence de Phlebotomus ariasi Tonnoir, 1921 en Tunisie et nouvelle mention de Phlebotomus chadlii Rioux, Juminer et Gibily, 1966. Archives de l'Institut Pasteur de Tunis, 1966, 3, 547551.

Croset H. Écologie et systématique des Phlebtomini (Diptera, Psychodidae) dans deux foyers, français et tunisiens de leishmaniose viscérale - Essai d'interprétation épidémiologique. Thèse Sciences Montpellier, 1969, 516 p.

Dancesco P., Ben Rachid M.S. \& Chadli A. Notes sur les Phlébotomes de la Tunisie. I. - Les espèces de Phlébotomes dans les collectivités urbaines et quelques aspects de leur écologie. Archives de l'Institut Pasteur de Tunis, 1968, 45, 177184.

Guerbouj S., Chemkhi J., Kâabi B., Rahali A., Ben Ismail R. \& GuIZAN I. Natural infection of the species Phlebotomus (Lar- roussius) langeroni (Diptera: Psychodidae) with Leishmania infantum in Tunisia. Transactions of the Royal Society of Tropical Medicine and Hygiene, sous presse.

Rioux J.A., Croset H., Léger N., Benmansour N. \& Cadi Soussi M. Présence de Phlebotomus bergeroti, Phlebotomus chabaudi, Phlebotomus chadlii et Sergentomyia christophersi au Maroc. Annales de Parasitologie Humaine et Comparée, 1975, 50, 493-506.

Rioux J.A., Guy Y., Le Coroller Y., Croset H. \& Addadi K. Présence en Algérie de Phlebotomus (Larroussius) chadlii Rioux, Juminer et Gibily, 1966. Bulletin de la Société de Pathologie Exotique, 1970, 63, 101-104.

Rioux J.A., Juminer B. \& Gibily H. Phlebotomus (Larroussius) chadlii n. sp. (Diptera-Psychodidae). Annales de Parasitologie Humaine et Comparée, 1966, 41, 83-89.

Reçu le 26 juillet 2006 Accepté le 30 août 2006 